ments have been based on femora taken from human skeletons, but it is now acknowledged that strontium lodges more permanently in the vertebrae than in the limbs. Although differences between the two measurements are small for young children, there may be considerable differences between the concentrations of strontium in adult bones from the two sources. In part, the Medical Research Council has been prompted to change from femora to vertebrae by the knowledge that measurements in countries other than Britain are usually based on vertebrae. Scotland will, however, continue with femora.

\section{Productive Physics}

AT the Rutherford Laboratory, the emphasis is on productivity. The laboratory houses Nimrod, the Science Research Council proton synchrotron, and the report for 1966 (HMSO, 15s.) is full of references to the availability of the machine and the hours of work completed. In view of the cost of the laboratory$£ 5.8$ million in the year ending March 31, 1966-this preoccupation is entirely understandable. Some of it derives, no doubt, from the frustrations of 1965 , when an alternator failure had Nimrod hobbling along at $2 \mathrm{GeV}$ for most of the year.

During 1966, Nimrod was back to its full power of $7 \mathrm{GeV}$, and operated for 4,850 hours at a mean beam intensity of $1.08 \times 10^{12}$ protons per pulse. Seven experiments with electronic techniques were completed, and two with bubble chambers. No significant $C$ (charge conjugation) violation was found in the decay $\eta \rightarrow \pi^{+} \pi-\pi^{0}$, so that the nature and origin of the $C P$ (charge conjugation $\times$ parity) violating interaction responsible for the decay $K_{L}{ }^{0} \rightarrow 2 \pi$ remain unknown. Rebuilding of the imposingly named British National Bubble Chamber continued during the year, and an $80 \mathrm{~cm}$ helium bubble chamber was commissioned. At the end of 1965-less than 2 years after Nimrod was first scheduled-it became apparent that the experimental hall was far too small, and approval to build a new hall was given at the end of 1966. This will allow more beams to be installed simultaneously, reducing the amount of modification needed for new experiments. The new hall, $300 \mathrm{ft}$. long by $150 \mathrm{ft}$. wide, will be completed by the end of 1968 .

The laboratory also houses a proton linear accelerator, which also had a record year for running in 1966. The PLA produces $50 \mathrm{MeV}$ protons, and has been used for experiments on proton scattering, pick-up reactions, and charge exchange reactions. During the year a variable energy cyclotron for AERE Harwell was completed, and the formal hand-over of control was to take place early in 1967 . This machine gives energies of $50 \mathrm{MeV}$ for protons and $80 \mathrm{MeV}$ for $\alpha$-particle beams. A new computer, an IBM 360/75, was obtained to replace Orion, and the laboratory continued to rely on the Chilton Atlas for the bulk of its off-line computing work. The Applied Physics Division did some work on superconducting magnets, and set up equipment to test superconducting cable up to 3,000 amps. The hope here is to replace conventional magnets with pulsed superconducting magnets, which would make future accelerators much smaller and cheaper. Similar techniques, the report hopefully suggests, could offer the opportunity of inexpensively converting Nimrod to an energy of $40-50 \mathrm{GeV}$.

\section{Field Reversals}

When Sir Edward Bullard delivered the 1967 Bakerian Lecture on the reversals of the Earth's magnetic field, he was introduced appropriately enough by Professor P. M. S. Blackett, President of the Royal Society. Between them, these two have shared much of the excitement of this intriguing field of research.

Sir Edward gave the reasons for believing that the magnetic field of the Earth has indeed reversed itself several times in the past. For him the conclusive evidence had been provided by the incredible agreement between the reversals derived from lavas and the linear anomalies observed in magnetic surveys of the ocean floor. The pattern of magnetization observed on the ocean floor was astonishingly symmetrical on either side of the mid-oceanic ridge, indicating that lava rises in the middle and flows away on either side. How did the field originate, and how could it reverse? Sir Edward lent his support to the double dynamo theory, involving two fields and two motionscomputer models of such arrangements had shown a pattern of reversals strikingly similar to the reversals of the Earth's field.

There were still two loose ends, Sir Edward thought. Among Colorado basalts, those deposited during periods of reversed magnetization were chemically different from those deposited during normal periods. "It seems inconceivable," he said, "that all reversed basalts are chemically different from all normal basalts." It was just possible that the direction of magnetization could affect the chemistry in some cases, but calculations had shown the effect to be very small. Was this, he wondered, something to do with ferromagnetism?

The other snag was provided by the disappearance of small animals called Radiolaria during reversals. It had been suggested that the absence of a field during the change-over had let in cosmic rays which caused mutations and eventually destroyed the species. Sir Edward was unhappy with this explanation. The increase in cosmic rays during a reversal was only about 10-15 per cent, he said, a small change compared with that observed between one latitude and another, or even in different depths of water. He left his audience with another suggestion: perhaps the reversal of the field lets in the solar wind, producing a thick ozone layer in the atmosphere. This might affect the atmosphere and the climate, and ultimately eliminate the Radiolaria. "It would be interesting," Sir Edward suggested, "to investigate magnetic field conditions at the time when dinosaurs disappeared."

\section{RNA Bacteriophage Replication from our Correspondent in Cell Biology}

LAST year two alternative models for the replication of the single-stranded RNA genomes of coliphage such as $\mathrm{Q} \beta, \mathrm{f} 2, \mathrm{MS} 2$ and R17 were hotly debated in journals and at conferences. One postulates that the parental RNA molecule (the plus strand) is replicated directly by a phage specific enzyme variously known as RNA polymerase, synthetase or replicase. The other postulates that an RNA molecule, the minus strand, complementary to the parental plus strand is synthesized first, giving rise to a double helical molecule with normal base-pairing, and this then acts as template for the semi- 